\title{
Robust Frequency-Adaptive PLL with Lyapunov Stability Guarantees
}

\author{
Gilberto Pin ${ }^{\mathrm{a}}$, Boli Chen ${ }^{\mathrm{b}}$, Giuseppe Fedele ${ }^{\mathrm{c}}$, Thomas Parisini ${ }^{\mathrm{d}}$
}

\begin{abstract}
The Global Quadrature Phase-Locked-Loop (GQPLL) is a recently-devised PLL-type architecture (based on Quadrature Signal Generation) able to track a biased sinusoidal signal with unknown frequency and amplitude. The original GQPLL formulation resorted to a non-standard nonnormalized adaptive law, able to guarantee the global convergence of the estimates for arbitrarily large adaptation gains, thus enabling arbitrarily fast adaptation transients. On the other side, the non-conventional adaptation law used in the original formulation makes it difficult to apply robustifying modifications of adaptive control. In this connection, the present work presents a new formulation for the PLL internal dynamics in order to obtain a convenient 1st order linear-in-the parameters error model, which can be dealt with by a conventional nonnormalized adaptive law, to which a robustifying modification such as projection can be applied. The large-gain global stability of the adaptive system is proven by Lyapunov arguments. The overall adaptive PLL is named Robustified GQPLL (RGQPLL).
\end{abstract}

\section{INTRODUCTION AND PROBLEM FORMULATION}

The present work concerns the design of an algorithm for tracking and estimating the unknown frequency of the following sinusoidal signal

$$
\begin{aligned}
& s(t)=A \sin \left(\vartheta(t)+\vartheta_{0}\right), \quad t \geq 0 \\
& \dot{\vartheta}(t)=\omega, \quad t \geq 0 \\
& \vartheta(0)=0,
\end{aligned}
$$

given the following biased/perturbed measurement

$$
y(t)=s(t)+c,
$$

where $c$ is an unknown scalar constant (usually referred to as "measurement bias" or "offset"). The fundamental frequency $\omega$, the amplitude $A$ and the initial angle $\vartheta_{0}$ of the sinusoid are unknown. We assume that $\omega$ is known to belong to the compact intervat: $\underline{\omega} \leq \omega \leq \bar{\omega}$, with $\underline{\omega}, \bar{\omega}: 0<\underline{\omega}<\bar{\omega}<+\infty$ finite bounds known (possibly) conservatively.

The problem of tracking sinusoidal signals with unknown frequency arises in many engineering domains, such as vibration attenuation in mechanical systems, acoustics, electrical power monitoring, signal processing and fault detection.

In the specific realm of power-systems engineering, PLLlike architectures are undisputedly the most used class of

a G. Pin is with the Dept. of Information Eng, Univ. of Padua, Italy (gilberto.pin@unipd.it);

b B. Chen is with the Dept. of Electrical and Electronic Engineering, Imperial College London, UK (boli.chen10@imperial.ac.uk);

G. Fedele is with the Dept. of Informatics, Modeling, Electronics and System Engineering, University of Calabria, Italy (giuseppe. fedeledunical.it);

d T. Parisini is with the Dept. of Electrical and Electronic Engineering, Imperial College London, UK. He's also with the Dept. of Engineering and Architecture, Univ. of Trieste, Italy and with the KIOS Research and Innovation Centre of Excellence, University of Cyprus, Cyprus (t.parisini@gmail.com). He has been partially supported by the European Union's Horizon 2020 Research and Innovation Programme under grant agreement No 739551 (KIOS CoE). algorithms used to track electrical signals, to synchronize inverters to the electrical grid and to assess the power quality (stability of voltage amplitude and grid-frequency). In case the frequency of the sinusoidal signal to be tracked is not perfectly known, such as in the case of "weak" grids, several modified PLL have been proposed to estimate the frequency. The recent work [1] offers a detailed overview of the most used architectures for single-phase applications, focusing in particular on those methods involving Quadrature Signal Generators (QSG). In the literature, QSG units are sometimes also referred to as Orthogonal Signal Generators (OSG). The resulting PLL schemes are commonly known as QSGPLLs or OSG-PLLs. The Frequency Locked-Loop (FLL) of [2], [3], that uses a Second Order Generalized Integrator (SOGI) to implement the OSG, is, for instance, capable of tracking sinusoidal signals with time-varying frequency and amplitude. Other methods providing frequency-adaptation ability to a PLL can be found in [4] and [5]. The Enhanced PLL (EPLL) of [6], [7] is a successful design that has found extensive application to the estimation of power-grid signal parameters. Compared to a conventional PLL, its output signal is locked both in phase and in amplitude with the input. While the original EPLL is not robust to the presence of DC-bias, several further modifications have been proposed to overcome this limitation. The reader is referred to [8] for a modified EPLL based on delayed-signal cancellation that features the rejection of DC-bias. The problem of rejecting the DC-offset has been addressed also in [9] and [10].

Abstracting from the aforementioned electrical and power systems applications, several solutions to the sinusoidal estimation problem outlined above have been conceived in the system-theoretic and signal processing fields, and many results are given in terms of adaptive observers (see [11], [12], [13], [14], [15], [16], [17] among others), or nonlinear adaptive systems [18], [19], [20]. Notably, all of these methods formally guarantee the global stability of the estimator. Despite coming along with appealing global stability guarantees, these methods are not widely used in applications, mainly due to their complexity compared to PLL.

On the contrary, the modified-PLL methodologies conceived and used in the electrical engineering domain, tailored for power-electrical applications and supported by strong experimental evidence, represent the preferred solution by the practitioners, even if supported just by local or smallgain stability proofs. Indeed, the formal stability proofs of SOGI-OSG-FLL and QSG-PLLs are based either on smallsignal analysis (local linearization of the dynamics), or averaging theory. The latter approach calls for unpractical smallgain assumptions on the adaptation gains. The small-gain 
assumption is also invoked by the stability proof of the EPLL scheme [6], which is based on variational arguments. As a final result, the adaptation rate for the estimated frequency must be tuned accurately to guarantee the stability of these algorithms in all the operating conditions. Typically, boosting the adaptation gains improves the convergence rate when the initial guess on the frequency is close to the true one and for clean measurements, but it may cause instability for large initialization error or in presence of measurement perturbations. In other words, global convergence may get lost in practice. In this connection, the contribution of the GQPLL scheme proposed in [21] is that of providing Lyapunov stability certificates by modifying a QSG-type PLL architecture through the introduction of auxiliary signal injections within the phase-locked loop.

In this work, the original GQPLL of [21] is modified in a threefold fashion:

- 1) A further filtered augmentation is introduced.

- 2) The auxiliary injection signals in the adaptive PLL are reformulated.

- 3) Points 1) and 2) allow to obtain, after non-trivial manipulations, an error-model of order 1 , that permits to use a conventional adaptation law with parameter projection.

The obtained frequency-adaptive sinusoid tracking system is named Robustified Global Quadrature PLL (RGQPLL).

\section{RGQPLL EQUATIONS}

Consider the equivalent trigonometric expression of the sinusoidal signal $s(t)$ :

$$
s(t)=a \sin (\vartheta(t))+b \cos (\vartheta(t)),
$$

where $a, b \in \mathbb{R}$ are unknown parameters related to the unknown amplitude $A$ of the sinusoid by $A=\sqrt{a^{2}+b^{2}}$. Letting $\hat{\Omega}(t)$ be the estimate of the squared-frequency $\Omega:=\omega^{2}$, we aim at tracking $y(t)=a \sin (\vartheta(t))+b \cos (\vartheta(t))+c$ by an estimated signal $\hat{y}(t)$ having the following structure:

$$
\hat{y}(t)=\hat{a}(t) \sin (\hat{\vartheta}(t))+\hat{b}(t) \cos (\hat{\vartheta}(t))+\hat{c}_{0}(t), t \geq 0
$$

where $\hat{c}_{0}(t)$ is a DC-bias compensation term, while the instantaneous estimated angle $\hat{\Omega}(t)$ evolves according to

$$
\dot{\hat{\vartheta}}(t)=\sqrt{\hat{\Omega}(t)}, t \geq 0
$$

with initial condition $\hat{\vartheta}(0)=0$ without loss of generality. The estimated squared-frequency must verify the lower and upper bounds $\underline{\Omega} \leq \hat{\Omega}(t) \leq \bar{\Omega}, \forall t \geq 0$, where $\underline{\Omega}:=\underline{\omega}^{2}$ and $\bar{\Omega}:=\bar{\omega}^{2}$ are known conservatively. The adaptation law for $\hat{\Omega}(t)$ will be presented in the next section.

The tracking objective can be attained by designing adaptation laws for $\hat{a}(t), \hat{b}(t), \hat{c}_{0}(t)$ and $\hat{\Omega}(t)$ such that $\lim _{t \rightarrow+\infty}|\hat{y}(t)-y(t)|=0$ and all the internal signals remain bounded. For the sake of notational simplicity, in the sequel we will neglect the explicit dependence from time of the signals and of the time-varying estimated parameters. In this Section, we provide the complete set of equations needed to implement the sinusoidal tracker/estimator. The reader can refer to Figure 1 for a block diagram representation of the RGQPLL.

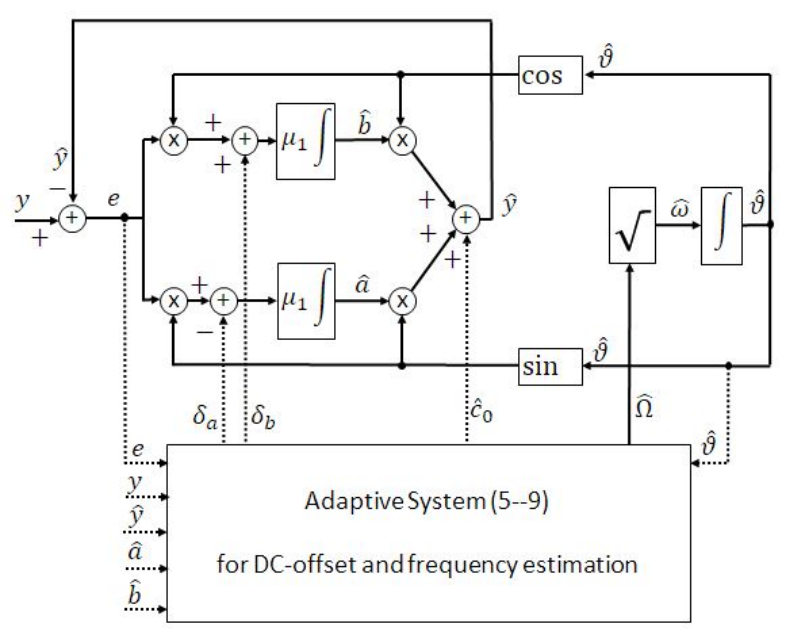

Fig. 1. Scheme of the RGQPLL. The dashed lines denote the additional signal paths compared to a conventional QSG-PLL. The improvements compared to the GQPLL of [21] are are concerned with the adaptive system, while the overall PLL-like structure is maintained.

In the sequel, we will denote by $e$ the tracking error:

$$
\begin{aligned}
e & :=y-\hat{y} \\
& =y-\hat{a} \sin \hat{\theta}-\hat{b} \cos \hat{\theta}-\hat{c}_{0} .
\end{aligned}
$$

Letting $\lambda_{0}>0$ and $\lambda_{1}>0$ be two arbitrary positive scalars, we will denote by $\mu_{0}$ and $\mu_{1}$ the scalar constants

$$
\mu_{0}:=\lambda_{0} \lambda_{1}, \mu_{1}: \lambda_{0}+\lambda_{1} .
$$

The equations for the online adaptation of $\hat{a}$ and $\hat{b}$ are given by the QSG-PLL-like multiplicative stage:

$$
\begin{aligned}
& \dot{\hat{a}}=\mu_{1} \sin (\hat{\vartheta}) e-\delta_{a}, \quad t \geq 0, \\
& \dot{\hat{b}}=\mu_{1} \cos (\hat{\vartheta}) e+\delta_{b}, \quad t \geq 0,
\end{aligned}
$$

with $\mu_{1}$ defined in (2), and where the signals $\delta_{a}$ and $\delta_{b}$ are suitable nonlinear injections aimed at stabilizing the dynamics of the RGQPLL. These signals are chosen as

$$
\begin{aligned}
& \delta_{a}:=\sqrt{\hat{\Omega}} \cos (\hat{\vartheta})\left(\eta_{0} \hat{c}_{0}+\eta_{1}\left(\hat{c}_{1}+\sqrt{\hat{\Omega}}(\hat{b} \sin (\hat{\vartheta})-\hat{a} \cos (\hat{\vartheta}))\right)\right), \\
& \delta_{b}:=\sqrt{\hat{\Omega}} \sin (\hat{\vartheta})\left(\eta_{0} \hat{c}_{0}+\eta_{1}\left(\hat{c}_{1}+\sqrt{\hat{\Omega}}(\hat{b} \sin (\hat{\vartheta})-\hat{a} \cos (\hat{\vartheta}))\right)\right),
\end{aligned}
$$

with $\hat{c}_{0}$ and $\hat{c}_{1}$ obtained through the second-order nonlinear filter:

$$
\begin{array}{lll}
\dot{\hat{c}}_{0} & =\hat{c}_{1}+\sqrt{\hat{\Omega}}(\hat{b} \sin (\hat{\vartheta})-\hat{a} \cos (\hat{\vartheta})), & t \geq 0 \\
\dot{\hat{c}}_{1} & =\left(\mu_{0}-\hat{\Omega}\right) e-\hat{\Omega} \hat{y}+\hat{K}-y_{1} \dot{\hat{\Omega}}+\lambda_{1}^{-1} \dot{\hat{K}}, \quad t \geq 0 \\
\hat{c}_{0}(0)=\overline{\hat{c}}_{0}, \quad \hat{c}_{1}(0)=\overline{\hat{c}}_{1}, &
\end{array}
$$

with $\mu_{0}$ defined in (2), while the auxiliary signal $y_{1}$ is obtained by filtering the output with the following 1 st order filter

$$
\begin{aligned}
& \dot{y}_{1}=-\lambda_{1} y_{1}+y, \quad t \geq 0 \\
& y_{1}(0)=\bar{y}_{1} .
\end{aligned}
$$

The signals $\eta_{0}$ and $\eta_{1}$ in (4) are defined as

$$
\begin{aligned}
& \eta_{0}:=1-\mu_{0} \frac{1}{\hat{\Omega}}, \\
& \eta_{1}:=-\mu_{1} \frac{1}{\hat{\Omega}}-\frac{\dot{\hat{\Omega}}}{2 \hat{\Omega}^{2}},
\end{aligned}
$$


where $\dot{\hat{\Omega}}$ is the derivative of the estimated squared-frequency to be described later on. This particular choice for the injection signals $\delta_{a}$ and $\delta_{b}$ and for the auxiliary signals $\eta_{0}$ and $\eta_{1}$ will be instrumental to obtain a 1 st order error model for the adaptive system. The initial conditions $\overline{\hat{c}}_{0}, \overline{\hat{c}}_{1}$ and $\bar{y}_{1}$ of the above filters can be set arbitrarily. Without loss of generality, we will assume $\overline{\hat{c}}_{0}=0, \overline{\hat{c}}_{1}=0$ and $\bar{y}_{1}=y(0)$.

Finally, the estimates for the auxiliary parameter $\hat{K}$ and for the squared-frequency $\hat{\Omega}$ are given by the constrained adaptation laws (the provided adaptation laws can be interpreted as a discontinuous projection of the parameter's derivatives to enforce the estimates into an admissible convex set):

$$
\dot{\hat{\Omega}}= \begin{cases}-k_{0} y_{1} e, \quad\left\{\begin{array}{c}
(\underline{\Omega}<\hat{\Omega}<\bar{\Omega}) \\
\vee((\hat{\Omega}=\underline{\Omega}) \wedge(e>0)) \\
\vee((\hat{\Omega}=\bar{\Omega}) \wedge(e<0)) \\
0,
\end{array}\right. \\
\left\{\begin{array}{c}
((\hat{\Omega}=\underline{\Omega}) \wedge(e \leq 0)) \\
\vee((\hat{\Omega}=\bar{\Omega}) \wedge(e \geq 0))
\end{array}\right.\end{cases}
$$

and

$$
\dot{\hat{K}}= \begin{cases}\lambda_{1}^{-1} k_{0} e, \quad\left\{\begin{array}{r}
(\underline{K}<\hat{K}<\bar{K}) \\
\vee((\hat{K}=\underline{K}) \wedge(e>0)) \\
\vee((\hat{K}=\bar{K}) \wedge(e<0)) \\
0,
\end{array}\right. \\
\left\{\begin{array}{c}
((\hat{K}=\underline{K}) \wedge(e \leq 0)) \\
\vee((\hat{K}=\bar{K}) \wedge(e \geq 0))
\end{array}\right.\end{cases}
$$

where $k_{0}>0$ is an arbitrary gain used to tune the adaptation speed, while $\underline{K} \in \mathbb{R}$ and $\bar{K} \in \mathbb{R}$ are the known (finite) lower and upper bounds for the angular speed. All in all, the RGQPLL has a total dynamical order of 8 with states $\hat{\Omega}, \hat{K}, \hat{c}_{0}, \hat{c}_{1}, \hat{a}, \hat{b}, \hat{\vartheta}$ and $y_{1}$.

\section{Stability ANALYSis}

Let us take the time-derivative of the error $e:=y-\hat{y}$ :

$$
\begin{aligned}
\dot{e} & =\omega a \cos (\vartheta)-\omega b \sin (\vartheta)-\dot{\hat{a}} \sin (\hat{\vartheta})-\hat{a} \sqrt{\hat{\Omega}} \cos (\hat{\vartheta}) \\
& -\dot{\hat{b}} \cos (\hat{\vartheta})+\hat{b} \sqrt{\hat{\Omega}} \sin (\hat{\vartheta})-\dot{\hat{c}}_{0} .
\end{aligned}
$$

Substituting the expression for $\dot{\hat{c}}_{0}$ then (10) is simplified into

$$
\dot{e}=\omega a \cos (\vartheta)-\omega b \sin (\vartheta)-\dot{\hat{a}} \sin (\hat{\vartheta})-\dot{\hat{b}} \cos (\hat{\vartheta})-\hat{c}_{1} .
$$

Then,substituting the expressions for $\delta_{a}$ and $\delta_{b}$ into (3) we get

$$
\begin{aligned}
& \dot{\hat{a}}=\mu_{1} \sin (\hat{\vartheta}) e-\sqrt{\hat{\Omega}} \cos (\hat{\vartheta})\left(\eta_{0} \hat{c}_{0}+\eta_{1} \dot{\hat{c}}_{0}\right), \\
& \dot{\hat{b}}=\mu_{1} \cos (\hat{\vartheta}) e+\sqrt{\hat{\Omega}} \sin (\hat{\vartheta})\left(\eta_{0} \hat{c}_{0}+\eta_{1} \dot{\hat{c}}_{0}\right)
\end{aligned}
$$

Finally, substituting the latter expressions into (11) we get the following intermediate result

$$
\begin{aligned}
\dot{e}= & \omega a \cos (\vartheta)-\omega b \sin (\vartheta)-\hat{c}_{1} \\
& -\mu_{1} \sin (\vartheta)^{2} e+\sin (\vartheta) \cos (\hat{\vartheta}) \sqrt{\hat{\Omega}}\left(\eta_{0} \hat{c}_{0}+\eta_{1} \dot{\hat{c}}_{0}\right) \\
& -\mu_{1} \cos (\hat{\vartheta})^{2} e-\cos (\hat{\vartheta}) \sin (\vartheta) \sqrt{\hat{\Omega}}\left(\eta_{0} \hat{c}_{0}+\eta_{1} \dot{\hat{c}}_{0}\right) \\
= & \omega a \cos (\vartheta)-\omega b \sin (\vartheta)-\hat{c}_{1} \\
& -\mu_{1} \sin (\vartheta)^{2} e-\mu_{1} \cos (\hat{\vartheta})^{2} e \\
= & \omega a \cos (\vartheta)-\omega b \sin (\vartheta)-\mu_{1} e-\hat{c}_{1} .
\end{aligned}
$$

The second time-derivative of the error yields:

$$
\ddot{e}=-\omega^{2}(y-c)-\mu_{1} \dot{e}-\dot{\hat{c}}_{1} .
$$

Now, substituting the expression for $\dot{\hat{c}}_{1}$ into (13) we obtain:

$$
\begin{aligned}
\ddot{e}= & -\Omega(y-c)-\mu_{1} \dot{e}+\hat{\Omega} e-\mu_{0} e+\hat{\Omega} \hat{y}-\hat{K}+y_{1} \dot{\hat{\Omega}} \\
& -\lambda_{1}^{-1} \dot{\hat{K}} \\
= & -\Omega y+\Omega c-\mu_{1} \dot{e}+\hat{\Omega} y-\mu_{0} e-\hat{K}+y_{1} \dot{\hat{\Omega}}-\lambda_{1}^{-1} \dot{\hat{K}} \\
= & (\hat{\Omega}-\Omega) y-(\hat{K}-K)-\mu_{1} \dot{e}-\mu_{0} e+y_{1} \dot{\hat{\Omega}}-\lambda_{1}^{-1} \dot{\hat{K}},
\end{aligned}
$$

where we have set $K:=\Omega c$. In view of this position, it turns out that the arbitrary bounds $\underline{K}$ and $\bar{K}$ can be set according to the a-priori (possibly) conservative information on the admissible range for the product $\Omega c$. Defining the parameter vector $\theta:=\left[\begin{array}{ll}\Omega & K\end{array}\right]^{\top}$, denoting its estimate by $\hat{\theta}:=\left[\begin{array}{ll}\hat{\Omega} & \hat{K}\end{array}\right]^{\top}$, and using the notation $\xi=\left[\begin{array}{ll}y & -1\end{array}\right]^{\top}$ for the regressors vector and $\xi_{1}:=\left[\begin{array}{ll}y_{1} & -\lambda_{1}^{-1}\end{array}\right]^{\top}$ for a vector of filtered regressors evolving according to:

$$
\dot{\xi}_{1}=-\lambda_{1} \xi_{1}+\xi
$$

with initial condition $\xi_{1}(0)=\left[y(0) \lambda_{1}^{-1}-\lambda_{1}^{-1}\right]^{\top}$, then we obtain the following expression for the dynamics of the tracking error:

$$
\ddot{e}=-\mu_{1} \dot{e}-\mu_{0} e+\xi^{\top}(\hat{\theta}-\theta)+\xi_{1}^{\top} \dot{\hat{\theta}} .
$$

Finally, defining the parameter error vector $\tilde{\theta}:=\theta-\hat{\theta}$, the tracking-error dynamics (15) can be re-arranged as

$$
\ddot{e}+\mu_{1} \dot{e}+\mu_{0} e=\xi^{\top} \tilde{\theta}+\xi_{1}^{\top} \dot{\hat{\theta}}
$$

In order to streamline the notation, in the following we will use the square-bracket shorthand commonly used in the adaptive control literature to denote the Laplace transform of a signal $u(\cdot): \mathbb{R} \rightarrow \mathbb{R}$

$$
\llbracket u \rrbracket(s)=\mathcal{L}\{u(\cdot)\}(s) .
$$

Transforming by Laplace both sides of (16) we can write

$$
\left(s^{2}+\mu_{1} s+\mu_{0}\right) \llbracket e \rrbracket(s)=\llbracket \xi^{\top} \tilde{\theta}+\xi_{1}^{\top} \dot{\hat{\theta}} \rrbracket(s),
$$

where we have neglected the contribution of the exponentially fading initial conditions (the polynomial $s^{2}+\mu_{1} s+\mu_{0}$ is Hurwitz by design). Now, in view of (2) we get the identity $s^{2}+\mu_{1} s+\mu_{0}=\left(s+\lambda_{0}\right)\left(s+\lambda_{1}\right)$, so that we can rewrite (17) as

$$
\llbracket e \rrbracket(s)=\frac{1}{\left(s+\lambda_{0}\right)\left(s+\lambda_{1}\right)} \llbracket \xi^{\top} \tilde{\theta}+\xi_{1}^{\top} \dot{\hat{\theta}} \rrbracket(s) .
$$

Letting

$$
\llbracket e_{1} \rrbracket(s):=\frac{1}{s+\lambda_{1}} \llbracket \xi^{\top} \tilde{\theta}+\xi_{1}^{\top} \dot{\hat{\theta}} \rrbracket(s)
$$

in the time domain we get

$$
e_{1}=\xi_{1}^{\top} \tilde{\theta}
$$

The latter expression can be proven either by invoking the Swapping Lemma or, directly, by differentiating $e_{1}$ :

$$
\begin{aligned}
\dot{e}_{1} & =\dot{\xi}_{1}^{\top} \tilde{\theta}+\xi_{1}^{\top} \dot{\hat{\theta}} \\
& =-\lambda_{1} \xi_{1}^{\top} \tilde{\theta}+\xi^{\top} \tilde{\theta}+\xi_{1}^{\top} \dot{\hat{\theta}} \\
& =-\lambda_{1} e_{1}+\xi^{\top} \tilde{\theta}+\xi_{1}^{\top} \dot{\hat{\theta}},
\end{aligned}
$$


thus obtaining the time-domain equivalent of (19). From (18) and (19) we obtain the expression

$$
\llbracket e \rrbracket(s)=\frac{1}{s+\lambda_{0}} \llbracket e_{1} \rrbracket(s),
$$

which finally yields

$$
\begin{aligned}
\dot{e} & =-\lambda_{0} e+e_{1} \\
& =-\lambda_{0} e+\xi_{1}^{\top} \tilde{\theta},
\end{aligned}
$$

that is a conventional 1st order error model that can be deal with by conventional adaptation laws, such as the projectionbased ones given in (8) and (9).

Studying the stability of equations (8), (9) and (20) alone is not sufficient, however, to characterize the stability of the overall adaptive system, which also consists of the linear filter (6) and of the nonlinear dynamics (5) (in charge of providing the signals $\hat{c}_{0}$ and $\hat{c}_{1}$ that are needed to compute the auxiliary injections $\delta_{a}$ and $\delta_{b}$ ). In this connection, let us take the second derivative of $\hat{c}_{0}$

$$
\begin{aligned}
\ddot{\hat{c}}_{0}= & \hat{\Omega}\left(\eta_{0} \hat{c}_{0}+\eta_{1} \dot{\hat{\hat{c}}}_{0}\right)+\left(\mu_{0}-\hat{\Omega}\right) e-\hat{\Omega} \hat{c}_{0}+\hat{K} \\
& +\frac{\dot{\hat{\Omega}}}{2 \sqrt{\hat{\Omega}}}(\hat{b} \sin (\hat{\vartheta})-\hat{a} \cos (\hat{\vartheta}))-y_{1} \dot{\hat{\Omega}}+\lambda_{1}^{-1} \dot{\hat{K}} \\
= & \hat{\Omega}\left(\eta_{0} \hat{c}_{0}+\eta_{1} \dot{\hat{\hat{c}}}_{0}\right)+\left(\mu_{0}-\hat{\Omega}\right) e-\hat{\Omega} \hat{c}_{0}+\hat{K} \\
& +\frac{\dot{\hat{\Omega}}}{2 \hat{\Omega}}\left(\dot{\hat{c}}_{0}-\hat{c}_{1}\right)-y_{1} \dot{\hat{\Omega}}+\lambda_{1}^{-1} \dot{\hat{K}} .
\end{aligned}
$$

Defining:

$$
v:=\left(\mu_{0}-\hat{\Omega}\right) e+\hat{K}-\frac{\dot{\hat{\Omega}}}{2 \hat{\Omega}^{2}} \hat{c}_{1}-y_{1} \dot{\hat{\Omega}}+\lambda_{1}^{-1} \dot{\hat{K}}
$$

and substituting in (21) the expressions for $\eta_{0}$ and $\eta_{1}$ given in (7), we obtain

$$
\begin{aligned}
\ddot{\hat{c}}_{0} & =\hat{\Omega}\left(\left(\eta_{0}-1\right) \hat{c}_{0}+\left(\eta_{1}+\frac{\dot{\hat{\hat{\Omega}}}}{2 \hat{\Omega}^{2}}\right) \dot{\hat{c}}_{0}\right)+v \\
& =\hat{\Omega}\left(\left(\eta_{0}-1\right) \hat{c}_{0}+\left(\eta_{1}+\frac{\dot{\hat{\Omega}}}{2 \hat{\Omega}^{2}}\right) \dot{\hat{c}}_{0}\right)+v \\
& =-\mu_{1} \dot{\hat{c}}_{0}-\mu_{0} \hat{c}_{0}+v .
\end{aligned}
$$

The latter differential equation describes the dynamics of the injection signal $\hat{c}_{0}$ and will be instrumental to analyze the internal stability of the adaptive PLL. The overall stability analysis will be performed in two steps, considering that the adaptive system can be viewed as composed by two subsystems, one cascaded to the other, with the first one being autonomous, so that it can be analyzed sequentially:

- A) the first autonomous system is made up of the tracking error dynamics (20) and the parameter-adaptation laws (8) and (9) (that correspond to the time-derivatives of the scalar components of the parameter vector $\hat{\theta}$ and, in turn, of the parameter error vector $\tilde{\theta}$ );

- $B$ ) the non-autonomous dynamical system (23) fed by $v$, defined in (22), that in turn depends on the tracking error and on the parameter estimates.

Step $A$ ): Consider now the following candidate Lyapunov function for the autonomous adaptive system (8), (9), (20) (recall that $\tilde{\theta}$ incorporates both the squared-frequency error $\tilde{\Omega}$ and $\tilde{K})$ :

$$
V=\frac{1}{2}\left(k_{0} e^{2}+\tilde{\theta}^{\top} \tilde{\theta}\right) .
$$

In this case, the stability analysis will be greatly simplified compared to the original GQPLL described in [21].

First, let us now make some comments on the saturated parameter adaptation law. Given the convexity of the admissible domain for the estimated parameters, the proposed saturated adaptation corresponds to a regular projection of $\hat{\theta} \in \mathbb{R}^{2}$ onto a closed rectangular domain. Thus, considering the properties of the projection operator in adaptive control (see [22]) for the sake of proving the semi-negativeness of the derivative of $V$ along the system's trajectory, it is sufficient to analyze the case in which the projection is non-active. Considering that $\dot{\tilde{\theta}}=\dot{\hat{\theta}}$, the derivative of $V$ along the system's trajectory writes:

$$
\begin{aligned}
\dot{V} & \leq k_{0} e \dot{e}+\tilde{\Omega} \dot{\hat{\Omega}}+\tilde{K} \dot{\hat{K}} \\
& =-\lambda_{0} k_{0} e^{2}+k_{0} e \xi_{1}^{\top} \tilde{\theta}-k_{0} \tilde{\Omega} y_{1} e+k_{0} \tilde{K} e \\
& =-\lambda_{0} k_{0} e^{2} .
\end{aligned}
$$

The convergence of the tracking error and the boundedness of the estimates can be proven by resorting to standard arguments of adaptive control based on the Lyapunov-like (Barbalat's) Lemma, [23]. Being $V$ bounded from below, non increasing and being $e$ uniformly continuous ( $\dot{e}$ is bounded), we have that $\lim _{t \rightarrow \infty} e=0$ and $\tilde{\theta}$ bounded. Moreover, considering that $y$ is bounded and that, in turn, $y_{1}$ is bounded, then $\lim _{t \rightarrow \infty} \dot{\hat{\theta}}=0$ (i.e., $\lim _{t \rightarrow \infty} \dot{\hat{\Omega}}=0$ and $\lim _{t \rightarrow \infty} \dot{\hat{K}}=$ $0)$. Invoking standard arguments of adaptive control, $\tilde{\theta}$ converges to zero (exponentially) in case the regressor's vector $\xi_{1}$ is persistently excited (PE), which is always the case for a non-degenerate sinusoid (non-zero amplitude and frequency), which implies that $\lim _{t \rightarrow \infty} \hat{\Omega}=\omega^{2}$ and $\lim _{t \rightarrow \infty} \hat{K}=K$.

Step $B)$ : It remains to prove the boundedness of the auxiliary signals $\delta_{a}, \delta_{b}, \hat{c}_{0}$ and $\hat{c}_{1}$. From equation (20) we can establish the boundedness of $\dot{e}$ (both $\tilde{\theta}$ and $\xi_{1}$ are bounded), then from (16) we can conclude that also $\ddot{e}$ is bounded. Considering that $e$ has a finite limit and $\ddot{e}$ is bounded (and therefore $\dot{e}$ is uniformly continuous), by Barbalat Lemma we also have that $\lim _{t \rightarrow \infty} \dot{e}=0$. This in turn implies (see last identity in (12)) that $\hat{c}_{1}$ is bounded and that $\lim _{t \rightarrow \infty} \hat{c}_{1}-(\omega a \cos (\vartheta)-\omega b \sin (\vartheta))=0$. This final result combined with (11) also implies that $\dot{\hat{a}}$ and $\dot{\hat{b}}$ are bounded. Moreover, the signal $v$ in (22) is bounded (it depends on bounded signals), then considering that the dynamics (23) are Hurwitz, we also have that $\hat{c}_{0}$ is bounded. Finally from (1) it follows that also $\hat{a}$ and $\hat{b}$ are bounded.

Remark 3.1: Note that the parameter projection is not mandatory to ensure the boundedness $\hat{a}$ and $\hat{b}$ : their boundedness can be inferred from that of other signals. The only parameters for which the projection is required (or other robustifying provisions such as $\sigma$-modification or deadzonemodification) are $\hat{\Omega}$ and $\hat{K}$, that appear in the Lyapunov function (24), whose time-derivative along system's trajectories is only semi-negative definite, in general.

In view of the above analysis, it turns out that the adaptation gain $k_{0}>0$ can be chosen arbitrarily large, enabling a fast convergence-rate while preserving global stability properties. The certifiable global stability of the RGQPLL 
in the nominal case, out of any small-gain assumption, represents a key benefit over the existent frequency-adaptive PLL schemes. Clearly, in case of measurement noise or unstructured perturbations (such as harmonics), the use of a large adaptation gain may amplify the disturbance, so that in practice the parameters should be tuned according to the specific application scenario.

At this point, we have proven that the estimated signal $\hat{y}(t)$ is able to track $y(t)$ with robust global stability guarantees, since the tracking error $e$ converges to zero even in absence of PE (corresponding to a degenerate sinusoidal input of zero amplitude or zero frequency). Now, in case of PE we would also like to provide an estimated for the unbiased sinusoid $s$. If the PE condition holds, have that, besides the signal boundedness and the asymptotic converge to zero of all the parameter derivatives to zero, also $\lim _{t \rightarrow \infty} \hat{K}=K$. By inspecting (22), it holds that $\lim _{t \rightarrow \infty} v=K$ and, since the non-autonomous dynamics of $\hat{c}_{0}$ (see (23)) is a stable LTI system whose input $v$ tends to a constant $\lim _{t \rightarrow \infty} v=K$, we have that $\lim _{t \rightarrow \infty} \hat{c}_{0}=K / \mu_{0}=\Omega c / \mu_{0}$. Choosing

$$
\hat{s}=\hat{y}-\mu_{0} \hat{c}_{0} / \hat{\Omega}
$$

then we obtain

$$
\begin{aligned}
s-\hat{s} & =y-\hat{y}+\mu_{0} \frac{\hat{c}_{0}}{\hat{\Omega}}-c \\
& =e+\mu_{0} \frac{\hat{c}_{0}}{\hat{\Omega}}-c,
\end{aligned}
$$

which finally yields

$$
\lim _{t \rightarrow \infty} s-\hat{s}=0
$$

We remark that, while the asymptotic convergence to zero of the tracking error $e$ has been proven also in absence of excitation, on the other hand the reconstruction of the unbiased sinusoid is guaranteed only in case of PE (nondegenerate input sinusoid). Moreover, we have not proven that $\hat{a}$ and $\hat{b}$ converge to $a$ and $b$, neither that they reach constant values; therefore these signals can be viewed as mere internal variables of the algorithm, instead of estimates of some time-invariant parameters. This is in line with the requirements of PLL schemes, that are usually required to track a sinusoid, filtering out noise, and, in this case, to estimate the its frequency while cancelling any measurement offset, if present.

\section{Simulation Results}

The behavior of the proposed RGQPLL is analyzed in this section through numerical simulations in a noisy scenario. For benchmarking purposes, the RGQPLL is compared against the EPLL of Kharimi-Garthemani [6], that is one of the most used architectures in electrical applications, the Adaptive Observer (AO) developed in [24], which is often taken as a baseline for comparison by the system-theoretic literature, and the original QPLL of [21].

The following sinusoidal signal is fed to the tracking algorithms, subjected to sudden frequency-variations:

$$
s(t)=320 \sin (2 \pi f t)
$$

with the measurement $y(t)=s(t)+c+d(t)$ corrupted by a random noise uniformly distributed in the interval $d(t) \in$ $[-10,10]$ where

$$
f=\left\{\begin{array}{ll}
52.5, & 0 \leq t<0.4 \\
47.5, & t \geq 0.4
\end{array} \quad, \quad c= \begin{cases}10, & 0 \leq t<1 \\
15, & t \geq 1\end{cases}\right.
$$

The parameters of the proposed RGQPLL are chosen as $\lambda_{0}=100, \lambda_{1}=400, k_{0}=1 e 9$. The admissible frequency range for the RGQPLL is set to $[25,100] \mathrm{Hz}$. For the sake of comparison, all the methods are initialized with the same guess $50 \mathrm{~Hz}$ for the frequency. Moreover, both the EPLL and the $\mathrm{AO}$ are tuned as in [21]. As shown in Fig. 2, the frequency change at $t=0.4 \mathrm{~s}$ is successfully detected by all the methods. The robustness of the RGQPLL is evident from the very small deviation at startup and from the accuracy of the estimate at steady state, that, on the other side, makes it recover slower from a frequency-change. Note that the slower convergence of the frequency estimate does not harm the signal reconstruction, which is faster than that achieved by GPLL. Indeed, the RGQPLL competes close to the EPLL in terms of tracking (that is, sinusoid denoising). A remarkable feature of the proposed algorithm, shared by GQPLL, can be appreciated at time $t=1 \mathrm{~s}$, when the DC-offset changes suddenly: while the RGQPLL estimate appears insensitive to this variation, instead the AO and the EPLL exhibit some oscillations. The tracking behaviour of each method is depicted in Fig. 3, from which it emerges clear that the RGQPLL shows remarkable noise immunity.

\section{Conclusions}

This work proposes a robust tracking algorithm for DCbiased sinusoidal signals with unknown frequency, amplitude and phase, named RGQPLL, bringing together the PLLlike architecture of QSG-PLL methods with the stability certificates of robust adaptive control with projection. The algorithm permits the complete rejection of the measurement DC-offset and enjoys the ability to track frequency variations.

\section{REFERENCES}

[1] S. Golestan, J. M. Guerrero, and J. C. Vasquez, "Single-phase PLLs: A review of recent advances," IEEE Trans on Power Electronics, vol. 32, no. 12, pp. 9013-9030, Dec 2017.

[2] G. Fedele and A. Ferrise, "A frequency-locked-loop filter for biased multi-sinusoidal estimation," IEEE Trans on Signal Processing, vol. 62 , no. 5, pp. 1125-1134, March 2014

[3] G. Fedele, A. Ferrise, and G. D'Aquila, "A global frequency estimator based on a frequency-locked-loop filter," in 2016 American Control Conference (ACC), July 2016, pp. 7001-7006.

[4] I. Carugati, P. Donato, S. Maestri, D. Carrica, and M. Benedetti, "Frequency adaptive PLL for polluted single-phase grids," IEEE Trans on Power Electronics, vol. 27, no. 5, pp. 2396-2404, May 2012.

[5] G. Pin, "A direct approach for the frequency-adaptive feedforward cancellation of harmonic disturbances," IEEE Trans on Signal Processing, vol. 58, no. 7, pp. 3523-3530, July 2010.

[6] M. Karimi-Ghartemani and M. R. Iravani, "A nonlinear adaptive filter for online signal analysis in power systems: applications," IEEE Trans on Power Delivery, vol. 17, no. 2, pp. 617-622, 2002.

[7] M. Karimi-Ghartemani, S. A. Khajehoddin, P. K. Jain, A. Bakhshai, and M. Mojiri, "Addressing DC component in PLL and notch filter algorithms," IEEE Trans on Power Electronics, vol. 27, no. 1, pp. 78-86, 2012.

[8] F. Wu, D. Sun, L. Zhang, and J. Duan, "Influence of plugging DC offset estimation integrator in single-phase ePLL and alternative scheme to eliminate effect of input DC offset and harmonics," IEEE Trans on Industrial Electronics, vol. 62, no. 8, pp. 4823-4831, Aug 2015. 


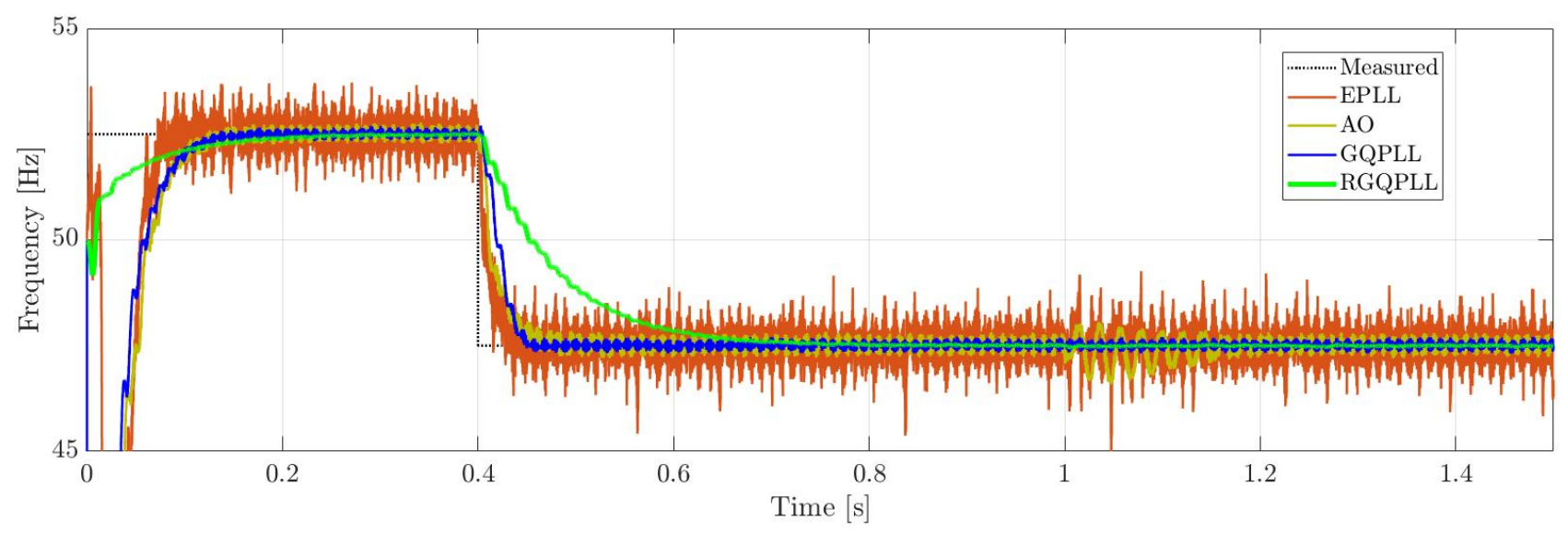

Fig. 2. Frequency estimates provided by the EPLL [7], the AO [24], the GQPLL [21] and the RGQPLL.

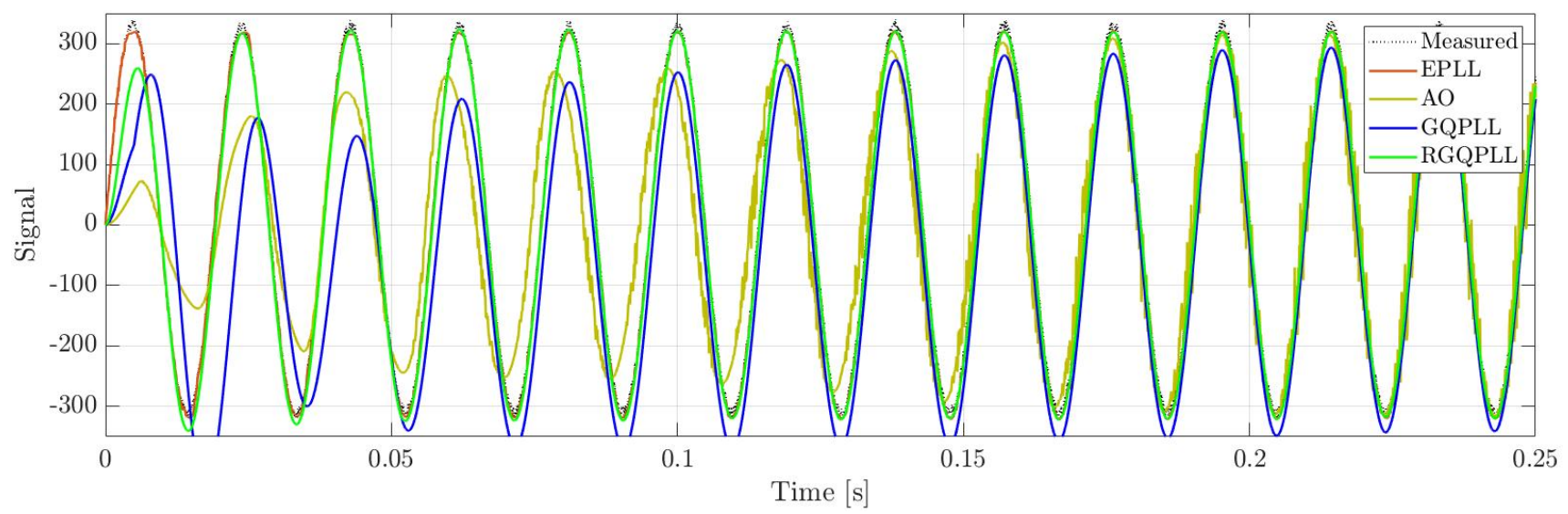

Fig. 3. Tracking performance of EPLL [7], AO [24], GQPLL [21] and RGQPLL.

[9] S. Hwang, L. Liu, H. Li, and J. Kim, "DC offset error compensation for synchronous reference frame PLL in single-phase grid-connected converters," IEEE Trans on Power Electronics, vol. 27, no. 8, pp. 3467-3471, Aug 2012.

[10] S. Golestan, M. Monfared, F. D. Freijedo, and J. M. Guerrero, "Design and tuning of a modified power-based PLL for single-phase grid-connected power conditioning systems," IEEE Trans on Power Electronics, vol. 27, no. 8, pp. 3639-3650, Aug 2012.

[11] M. Hou, "Estimation of sinusoidal frequencies and amplitudes using adaptive identifier and observer," IEEE Trans on Automatic Control, vol. 52, no. 3, pp. 493-499, March 2007.

[12] A. A. Bobtsov, N. A. Nikolaev, O. V. Slita, A. S. Borgul, and S. V. Aranovskiy, "The new algorithm of sinusoidal signal frequency estimation," IFAC Proceedings Volumes, vol. 46, no. 11, pp. 182 - 186, 2013, 11th IFAC Workshop on Adaptation and Learning in Control and Signal Processing.

[13] S. Aranovskiy, A. Bobtsov, A. Kremlev, N. Nikolaev, and O. Slita, "Identification of frequency of biased harmonic signal," European J. of Control, vol. 16, no. 2, pp. 129 - 139, 2010.

[14] B. Chen, G. Pin, W. M. Ng, C. K. Lee, S. Y. R. Hui, and T. Parisini, "An adaptive observer-based switched methodology for the identification of a perturbed sinusoidal signal: Theory and experiments," IEEE Trans on Signal Processing, vol. 62, no. 24, pp. 6355-6365, Dec 2014.

[15] B. Chen, G. Pin, W. M. Ng, S. Y. R. Hui, and T. Parisini, "An adaptive-observer-based robust estimator of multi-sinusoidal signals," IEEE Trans on Automatic Control, vol. 63, no. 6, pp. 1618-1631, June 2018.

[16] G. Pin, Y. Wang, B. Chen, and T. Parisini, "Identification of multisinusoidal signals with direct frequency estimation: An adaptive observer approach," Automatica, vol. 99, pp. 338 - 345, 2019.

[17] B. Chen, P. Li, G. Pin, G. Fedele, and T. Parisini, "Finite-time estimation of multiple exponentially-damped sinusoidal signals: A kernel-based approach," Automatica, vol. 106, pp. 1 - 7, 2019.
[18] G. Pin, B. Chen, T. Parisini, and M. Bodson, "Robust sinusoid identification with structured and unstructured measurement uncertainties," IEEE Trans on Automatic Control, vol. 59, no. 6, pp. 1588-1593, June 2014.

19] B. Chen, G. Pin, W. M. Ng, S. Y. R. Hui, and T. Parisini, "A parallel prefiltering approach for the identification of a biased sinusoidal signal: Theory and experiments," Int. J. Adaptive Control and Signal Proc., vol. 29, no. 12, pp. 1591-1608, 2015

[20] G. Pin, B. Chen, and T. Parisini, "Robust finite-time estimation of biased sinusoidal signals: A volterra operators approach," Automatica, vol. 77 , pp. $120-132,2017$.

[21] G. Pin, B. Chen, G. Fedele, and T. Parisini, "Globally-stable tracking and estimation for single-phase electrical signals with dc-offset rejection," in IECON 2019 - 45th Annual Conference of the IEEE Industrial Electronics Society, vol. 1, Oct 2019, pp. 4663-4668.

[22] E. Lavretsky and T. E. Gibson, "Projection operator in adaptive systems," ArXiv, vol. abs/1112.4232, 2012.

[23] J. Slotine and W.Li, Applied Nonlinear Control. Pearson, 1990.

[24] M. Hou, "Parameter identification of sinusoids," IEEE Trans on Automatic Control, vol. 57, no. 2, pp. 467-472, 2012. 